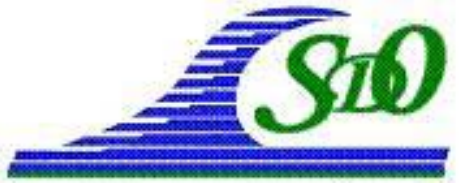

XI ${ }^{\text {èmes }}$ Journées Nationales Génie Côtier - Génie Civil

Les Sables d'Olonne, 22-25 juin 2010

DOI:10.5150/jngcgc.2010.090-R

(C) Editions Paralia CFL

disponible en ligne - http://www.paralia.fr - available online

\title{
Le projet SEM-REV : un site d'expérimentation en mer pour la recherche et l'industrie
}

\author{
Jean-Marc ROUSSET ${ }^{1}$, Hakim MOUSLIM ${ }^{1}$, \\ Gérard LE BIHAN ${ }^{1}$, Aurélien BABARIT ${ }^{1}$
}

\author{
1. Equipe Hydrodynamique et Génie Océanique, LMF UMR 6598, \\ Ecole Centrale de Nantes, 1 rue de la Noë, 44321 Nantes, France. \\ jean-marc.rousset@ec-nantes.fr
}

\section{Résumé :}

SEM-REV est un projet visant à mettre en place un site d'expérimentation en mer pour les systèmes houlomoteurs de production d'électricité. Constitué d'une zone en mer au large du Croisic, d'une base à terre et de liaisons électriques, ce projet est unique sur le littoral français. Il s'agit de pouvoir tester dans des conditions normales d'opérations des systèmes de conversion de l'énergie des vagues en connaissant le mieux possible les conditions environnementales du site. Les principaux objectifs techniques et scientifiques sont exposés ainsi que sont décrites les principales étapes du développement du projet. Des précisions sont données concernant les démarches administratives exigées par les autorités. Une partie de l'instrumentation de terrain est également présentée.

Mots-clés :

Energies marines - Expérimentation - Mesures - Etudes d'impact

\begin{abstract}
:
SEM-REV is an innovative project leading to establish a test zone for Wave Energy Converters (WEC) on the French Atlantic Coast. The project is composed of a field area offshore Le Croisic to test the machines, some dedicated buildings onshore for data analyses and technical purposes, electrical components in order to connect the WEC to the power grid. The major technical and scientific objectives are described, as well as significant phases of the project development regarding permitting processes. Environmental conditions are measured by meteo-oceanographical equipments which are described in the paper.
\end{abstract}

\section{Introduction}

Le projet SEM-REV est un projet visant à mettre en place, au large de la presqu'île de Guérande, un Site d'Expérimentation en Mer dédié aux dispositifs Récupérateurs de l'Energie des Vagues ou dispositifs houlomoteurs. L'objectif majeur est de tester des machines en condition opérationnelle en vue de leur mise au point. Le projet est unique en France et devrait débuter sa phase d'exploitation mi-2010. 
D'autres projets de zones d'expérimentation en mer sont en développement en Europe. A l'heure actuelle, seul le site écossais EMEC (aux îles Orcades) est opérationnel. Plusieurs autres sites (PPZ au Portugal, BIMEP au Pays Basque, WaveHub au Royaume Uni) sont à divers niveaux d'aboutissement avec souvent des soucis de financements.

SEM-REV est issu des activités de l'Ecole Centrale de Nantes dans les énergies marines renouvelables et développé par l'équipe Hydrodynamique et Génie Océanique du Laboratoire de Mécanique des Fluides (UMR 6598 du CNRS). Le projet se compose de plusieurs éléments complémentaires :

- une zone en mer qui accueille les systèmes houlomoteurs en essai et sur laquelle sont déployés des dispositifs de mesures ;

- une base à terre pour la réception des données transmises à partir du site en mer et abriter des moyens techniques ;

- un raccordement au réseau métropolitain de transport de l'électricité par un câble électrique sous-marin et un poste de livraison à terre ;

- des zones logistiques pour le déploiement des machines en mer et leur maintenance.

Les objectifs scientifiques du site d'expérimentation dans le domaine de l'énergie des vagues sont les suivants (MOUSLIM et al. 2008) :

- évaluer et améliorer la performance des prototypes ;

- mettre au point les composants et les sous-systèmes ;

- analyser l'interaction avec le réseau électrique ;

- définir un protocole d'analyse de l'impact environnemental.

D'autres activités liées au secteur du génie océanique vont être développées grâce à la présence pérenne de l'instrumentation en mer : essais en mer de petits navires inférieurs à $100 \mathrm{~m}$, essais en mer de modèles réduits (navires, structures offshore) de dimensions supérieures à celles des maquettes testées en bassin (notamment ceux de Centrale Nantes), mesures et validation de la prédiction des états de mer court et long terme.

\section{Description du projet}

Débuté en octobre 2007 et financé majoritairement par le Contrat de Projets Etat Région des Pays de la Loire (CPER 2007 - 2013), ce projet poursuit sa mise en place et devrait pour partie être opérationnel à l'été 2010.

\subsection{La zone d'essais en mer}

La zone en mer est située à une douzaine de milles marins au large du Croisic. Comme le montre la figure 1, elle est légèrement à l'Ouest d'une ligne partant du plateau du Four jusqu'à celui de la Banche (figure 1). Le site sera prochainement déclaré zone d'utilisation exclusive sur environ $1 \mathrm{~km}^{2}$ et sera alors matérialisé à ses 4 coins par un balisage effectué avec le concours du CETMEF / Service des Phares et Balises.

La profondeur moyenne sur le site est d'environ $35 \mathrm{~m}$, le marnage maximal étant de 5,5 m. Les conditions océano-météo permettent de compter sur des courants marins d'au 


\section{XI ìmes Journées Nationales Génie Côtier-Génie Civil}

Les Sables d'Olonne, 22-25 juin 2010

plus 1 nœud (SO - NE) avec des vents dominants de Sud-Ouest (70\% d'occurrence entre $240^{\circ}$ et $260^{\circ}$ ).

Les conditions de houle sont modérées avec 177 jours par an pendant lesquels la hauteur significative est supérieure à $1 \mathrm{~m}$. La puissance disponible d'énergie houlomotrice est estimée à $12 \mathrm{~kW} / \mathrm{m}$ en moyenne annuelle (figure 2).

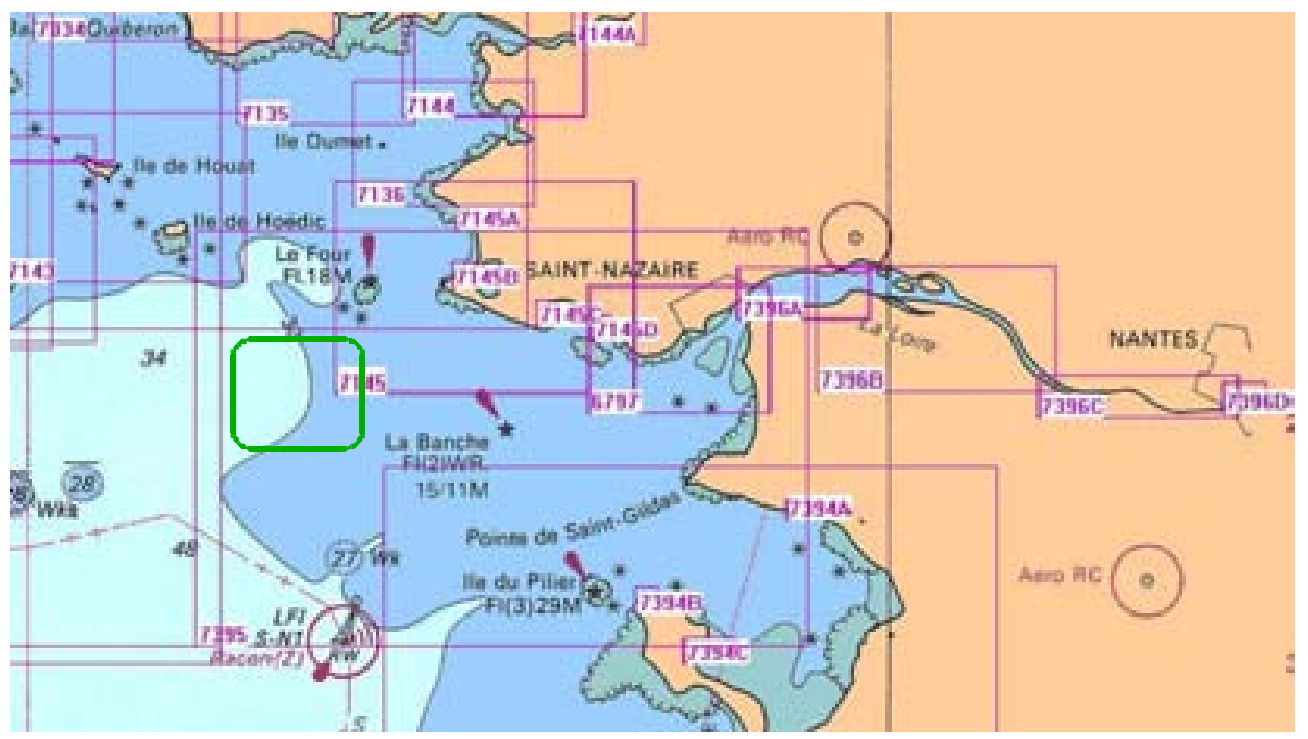

Figure 1. Localisation du site SEM-REV au large du Croisic.

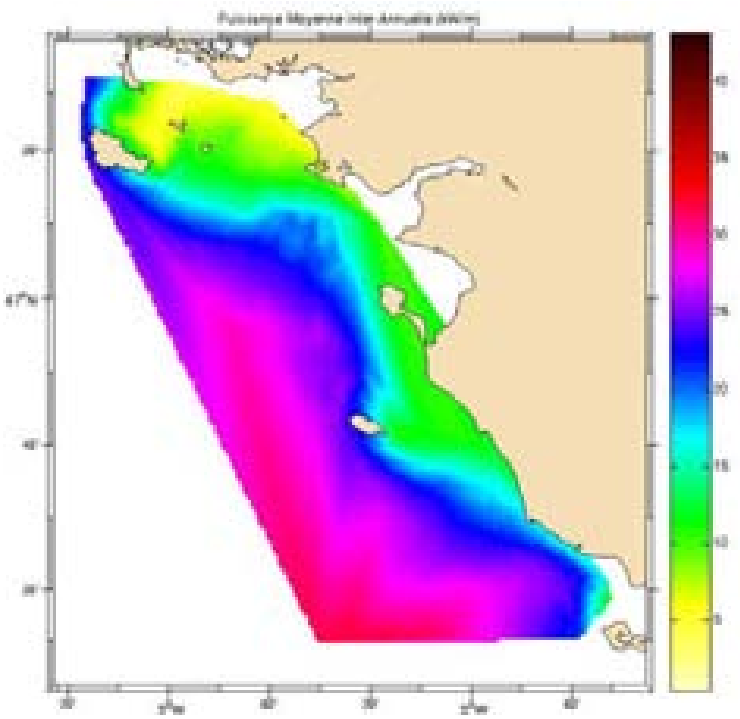

Figure 2. Carte d'estimation de la ressource houlomotrice annuelle en $\mathrm{kW} / \mathrm{m}$.

\subsection{La base à terre}

La base de recherche à terre est située sur le site de Pen Avel, sur la commune du Croisic, le terrain appartenant au Conservatoire du Littoral. Près de $200 \mathrm{~m}^{2}$ de bureaux, salles de réunion etc. sont déjà disponibles dans une ancienne villa réaménagée il y a 


\section{Thème 6 - Energies marines}

plusieurs années. Des travaux de modifications sont en cours afin de transformer un bâtiment annexe en locaux dédiés à l'accueil du personnel opérant le site (espace de restauration, ateliers, couchages temporaires). Ces locaux vont également servir à abriter la centrale de réception des données.

Ce dernier objectif est stratégique car il recouvre les activités liées à la centralisation et l'archivage des données, le contrôle en temps réel des différents éléments du site avec la gestion des alarmes et des sécurités.

La base à terre sert ainsi de relais vis à vis de l'extérieur pour tous les aspects scientifiques, automatiques et électriques, la totalité des informations et des mesures aboutissant ici :

- les données environnementales de la zone en mer ;

- les données et dispositifs de contrôle (SCADA) de la machine en test ;

- les données et les dispositifs de contrôle de la production électrique.

\subsection{Le raccordement électrique}

Un câble électrique va être prochainement mis en place entre la zone d'essais en mer et le poste de livraison électrique où s'effectue le raccordement au réseau de distribution haute tension (HTA). Ce câble sous-marin est conçu pour une puissance de 2,5 MVA et $20 \mathrm{kV}$. Différents composants sous-marins vont également être mis en place afin de permettre la (dé)connexion entre la machine et le câble.

Plusieurs campagnes de bathymétrie et de reconnaissance des fonds ont permis de déterminer des routes possibles du câble en tenant compte de multiples contraintes parmi lesquelles : sa longueur (donc son coût), la difficulté technique de sa tenue sur des zones exposées (existences de platiers rocheux à contourner etc.), les zones de conflits avec les autres usages de la mer. La pose du câble est programmée début 2011.

Le raccordement terrestre au réseau de distribution électrique $20 \mathrm{kV}$ de ERDF est effectué par un poste de livraison qui contient plusieurs composants pour le contrôle, la surveillance et la validation de la qualité de l'électricité produite ainsi que la protection de tout le système. Il représente un des points clés du projet SEM-REV car il donne la possibilité d'étudier les interactions entre une machine productrice d'énergie marine renouvelable et le réseau réel de distribution électrique.

\subsection{Les moyens et les zones logistiques}

La proximité géographique du port de St Nazaire est un atout dans le projet SEM-REV. En effet le Grand Port Maritime de Nantes Saint-Nazaire (4 ${ }^{\text {ème }}$ port français) est doté d'importantes capacités logistiques par ses bassins et ses moyens portuaires (remorqueurs, grues etc.), ses quais et entrepôts mais également par la présence de nombreux sous-traitants spécialisés. Ces différents éléments sont aptes à fournir l'assistance nécessaire à l'assemblage, le déploiement et la maintenance des machines et des équipements associés (hydraulique, électricité etc.). Ces moyens lourds seront 


\section{XI $I^{\text {èes }}$ Journées Nationales Génie Côtier - Génie Civil Les Sables d'Olonne, 22-25 juin 2010}

notamment mis à contribution pour la mise en place des éléments d'ancrage nécessaires aux convertisseurs.

\section{Implantation et démarrage du projet}

La recherche d'un site d'essai adapté pour la mise au point de prototypes de systèmes de récupération d'énergie des vagues a été effectuée sur la base de nombreux critères tenant compte des aspects techniques, fonciers, réglementaires et environnementaux.. Les études de faisabilité ont dégagé plusieurs critères d'arbitrage parmi lesquels : le potentiel énergétique de vagues, le raccordement au réseau électrique, les servitudes réglementaires et environnementales et les coûts d'investissement. La mise en place d'un Système d'Information Géographique dédié a permis d'identifier les zones potentielles d'incompatibilité en raison de la navigation, des activités de pêches, des raisons militaires ou archéologiques.

Des procédures de consultations ont été lancées à partir d'une phase initiale d'identification des partenaires qui a conduit à définir trois catégories de parties prenantes (tableau 1) : des autorités administratives, des partenaires stratégiques avec lesquels le projet doit se monter, des partenaires locaux sans lesquelles le projet ne peut être accepté.

Tableau 1. Parties prenantes impliquées dans la procédure de consultations.

\begin{tabular}{ll}
\hline Catégorie & Partenaires \\
\hline \multirow{2}{*}{ Autorités } & Ministère de l'Ecologie, de l'Energie, du Développement durable et de la Mer \\
administratives & Affaires Maritimes (administration nationale) \\
& Conseil Régional des Pays de la Loire \\
& Conseil Général de Loire Atlantique \\
\hline Partenaires & Direction Régionale des Affaires Maritimes \\
stratégiques & Conservatoire du Littoral \\
& Electricité Réseau Distribution de France \\
& Directions régionales de l'Environnement \\
\hline & Mairies de la presqu'île de Guérande \\
& Résidents des communes concernées \\
& Association de pêcheurs, d'activités nautiques etc. \\
& Clubs de plongée et clubs de voile
\end{tabular}

Un noyau restreint parmi lesquels figuraient plusieurs partenaires clés a été formé afin d'assurer une communication efficace au cours des procédures de consultation. Ce comité technique a été mis à contribution à plusieurs pour différentes questions environnementales, techniques ou administratives. Un plan global d'information a été 
mis en place dès le début afin de faire connaître à tous les partenaires, et de façon régulière, l'état d'avancement du projet, y compris sur les aspects techniques.

\section{Evaluations environnementales}

SEM-REV est un projet pionnier en France vis-à-vis de la génération d'énergies marines renouvelables. Il est apparu rapidement que la législation de l'environnement ne comportait pas d'exigences spécifiques à ce type de projet (HUERTAS-OLIVARES et al., 2007) mais plusieurs procédures se recouvraient.

De façon synthétique, les demandes d'autorisations nécessaires à la mise en place du site en mer se font dans le cadre (non exclusif) de: la directive 85/337/CEE (Environmental Impact Assessment)sur les études d'impacts environnementaux (, les directives Oiseaux (79/409/CEE) et Habitats (92/43/CEE) à l'origine du réseau Natura $2000 »$ regroupe des zones naturelles protégées, le Code de l'Environnement qui précise le contenu, le déroulement et les objectifs des études d'impact.

Les évaluations environnementales se décomposent alors en plusieurs parties :

- Un état initial qui s'intéresse à la zone dans laquelle serait implanté le site, du point de vue du milieu naturel, des ressources marines et littorales, des activités de loisirs et touristiques entre autres.

- Une évaluation des effets directs et indirects induits par les modifications (temporaires ou permanentes) du milieu naturel dans le cadre du projet. Cette analyse s'intéresse en particulier aux points suivants : l'environnement physique, les écosystèmes, les paysages, les aspects sociaux, la sûreté, les impacts sur la santé, les usages divers à terre et en mer.

- Une justification des différents éléments du projet en terme de spécifications, dispositions géographiques et de programmation des infrastructures.

- Une présentation des mesures de réduction (voire de compensation) des conséquences éventuelles du projet sur l'environnement et la santé. L'aspect économique des ces mesures doit impérativement être traité.

- Une analyse de la méthodologie envisagée pour le suivi environnemental.

Ces études représentent des jalons décisifs dans l'avancement du projet SEM-REV. Elles sont maintenant terminées et la procédure d'obtention des autorisations est en cours d'achèvement.

\section{Procédures d'obtention des autorisations}

Les agréments nécessaires à l'installation de prototypes à l'échelle 1, même pour une zone d'essais, ne s'obtiennent qu'après avoir suivi la procédure actuelle concernant les projets de production d'énergie en mer. Elle aboutit à la délivrance de trois types d'autorisations :

- un permis d'exploitation délivré par le Ministère en charge de l'Energie ;

- une concession temporaire pour l'occupation sur une zone déterminée du domaine 


\section{XI $I^{\grave{m} e s}$ Journées Nationales Génie Côtier - Génie Civil \\ Les Sables d'Olonne, 22-25 juin 2010}

marin ;

- un agrément vis à vis de la Loi sur l'Eau.

D'autres dispositions légales doivent être également respectées pour les infrastructures en zone littorale, comme les permis de construire pour les bâtiments abritant la base à terre par exemple.

Toutes ces démarches administratives (évaluations et autorisations) font appel à de nombreux partenaires clés dont les points de vue initiaux ne sont pas tous concordant. Les efforts d'analyse et de concertation paient néanmoins et les autorisations devraient être promulguées très prochainement.

La production d'énergie électrique et le raccordement au réseau de distribution demandent des autorisations spécifiques qui sont essentiellement délivrées par ERDF. La procédure recouvre notamment trois points: un permis d'exploitation, une autorisation de raccordement électrique et un accord de rachat de la production électrique.

L'autorisation de raccordement découle d'une évaluation initiale du projet conduite par ERDF sur la base des spécifications électriques et techniques présentées par l'Ecole Centrale de Nantes. Cette étude détaillée vérifie la conformité du projet aux différentes exigences liées au réseau de distribution. Ses conclusions amènent alors à une proposition technique et financière.

L'évaluation sur le raccordement au réseau est faite à partir de l'analyse électrique des systèmes de production et de l'impact potentiel de cette production sur le réseau. La qualité de l'énergie électrique doit être respectée en termes de génération d'harmoniques, de fluctuations de voltage, de puissance réactive, de montée et d'écroulement de la tension, etc.

La modélisation du système de production conduit à établir des bornes entre lesquelles les développeurs devront demeurer pour être en accord avec les autorisations accordées à SEM-REV. Il est en effet nécessaire à ERDF d'estimer le comportement des machines pour différents niveaux de charge (jusqu'à 2,5 MVA) ce qui peut conduire à exiger la mise en place d'équipements spécifiques pour le contrôle et la régulation de la fréquence et de la tension électrique.

La plupart de ces procédures et autorisations sont faites pour le projet de site en mer et les dispositifs houlomoteurs ne sont pas concernés par beaucoup d'entre elles. Toutefois l'accueil d'une machine ne sera possible que si elle satisfait aux critères techniques et environnementaux énoncés dans le cadre de ces autorisations.

\section{Evaluation de la performance des machines}

Schématiquement la performance des dispositifs houlomoteurs est évaluée à partir de la connaissance des conditions environnementales et des caractéristiques électriques. 


\subsection{Les mesures en mer}

Le site en mer est équipé d'un certain nombre de capteurs et d'équipements afin de caractériser les conditions environnementales en temps réel. Les données recueillies sont analysées par les outils du LMF puis archivées et / ou diffusées.

Le site SEM-REV permet un enregistrement en continu de données terrain et ainsi différents niveaux d'analyse : prévisions court terme (quelques minutes) par des calculs en temps réel, prévisions à moyen terme d'états de mer et d'énergie houlomotrice.

En particulier les données des différents capteurs permettent de travailler sur la prédiction déterministe et temporelle de la surface libre élargissant ainsi le champ des travaux de recherche conduits dans le bassin de génie océanique de l'ECN.

Les équipements scientifiques financés par le projet et déployés sur le site en mer sont les suivants (figure 3 ) :

- 2 bouées de mesure de houle directionnelle Datawell WaveRider ;

- 2 courantomètres acoustiques immergés ADCP RDI WorkHorse Sentinel ;

- 1 bouée météo dotée de nombreux capteurs pour la mesure du vent, de la température, de l'humidité, du rayonnement solaire direct et indirect, ... .

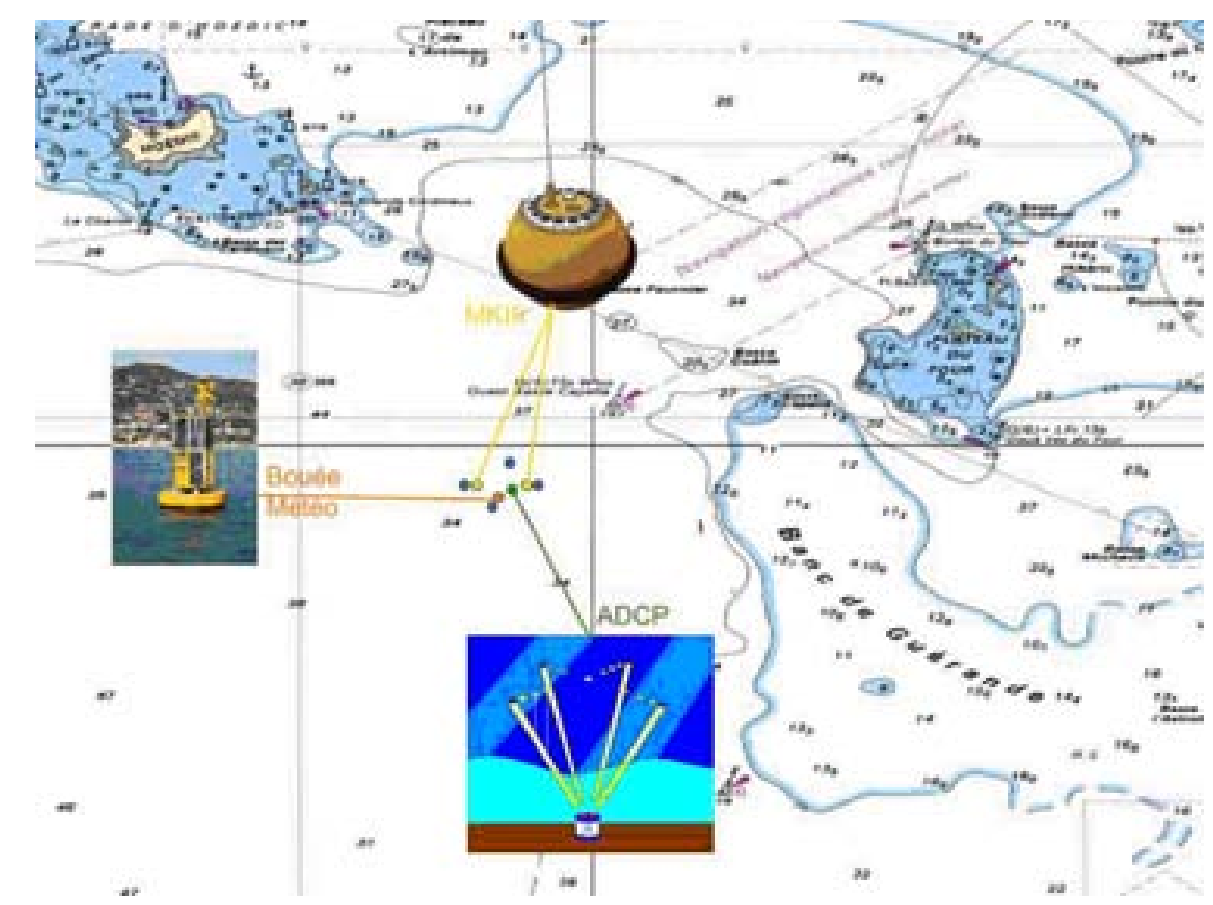

Figure 3. Localisation schématique de l'instrumentation sur le site.

Le déploiement a débuté à l'automne 2009 et va se poursuivre ce printemps 2010. La collaboration mise en place avec le CETMEF donne l'opportunité de mouiller une troisième bouée Datawell qui elle sera un peu plus au large. Une station légère pour la mesure du vent devrait être installée sur le phare du Four et plusieurs stations terrestres de Météo-France complèteront les données atmosphériques. 


\section{XI $I^{\text {èmes }}$ Journées Nationales Génie Côtier - Génie Civil Les Sables d'Olonne, 22-25 juin 2010}

Outre le nombre d'instruments installés sur une zone en mer finalement assez restreinte, plusieurs particularités font de SEM-REV un site remarquable. En effet le projet a l'ambition de faire que les équipements mesurent en continu tout au long de l'année et que la plupart transmettent également en temps réel les données temporelles.

Par exemple les données de houle transmises à terre par les bouées sont constituées des statistiques et caractéristiques spectrales usuelles (hauteurs significatives, périodes, direction) mais les élévations temporelles de la surface libre peuvent également être transmises. Ces mesures sont alors analysées à l'aide de codes numériques qui permettent une bonne séparation et identification des systèmes énergétiques qui composent ces états de mer complexes (KERBIRIOU et al., 2007).

La livraison des différentes bouées a donné l'opportunité de les soumettre à des essais préalables en bassin de génie océanique (longueur $50 \mathrm{~m}$, largeur $30 \mathrm{~m}$ et profondeur $5 \mathrm{~m}$, Hs maxi $0,70 \mathrm{~m}$ ) afin de tester les dispositifs d'acquisitions, les communications et les méthodes d'analyse des données. Deux vues des essais de la bouée météo et d'une bouée de houle sont présentées en figure 4 .
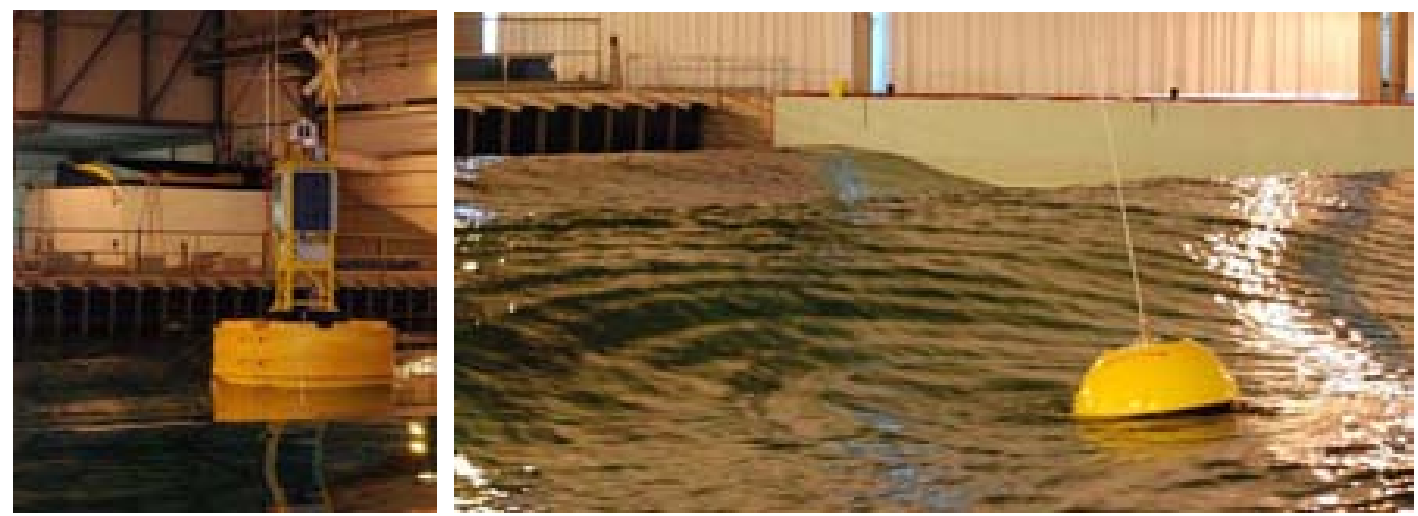

Figure 4. Essais en bassin de la bouée météo (gauche) et d'une bouée Datawell (droite).

\subsection{La puissance électrique d'une machine}

La puissance électrique produite par un système houlomoteur est évaluée au niveau du poste de livraison. La qualité du courant et le flux instantané d'énergie étant des caractéristiques variant avec le temps, elles peuvent être analysées en regard des données temporelles de l'environnement recueillies simultanément (houle, vent, etc.). Le dispositif de conversion de l'énergie des vagues (PTO, power take off) peut ainsi être étudié dans les conditions réelles d'exploitation. En particulier trois états électriques seront à examiner au travers de la mesure en continu du voltage et de la fréquence du courant : états nominal, état transitoire et état endommagé. 


\section{Conclusion}

Le site d'expérimentation en mer pour les systèmes houlomoteurs de production d'énergie SEM-REV va être opérationnel au cours de l'été 2010 pour la partie mesures océano-météo sur site. Les phases de développement se poursuivent avec la délivrance prochaine des différentes autorisations quant à l'usage d'une zone balisée en mer, à la pose du câble électrique et au raccordement au réseau de distribution.

La pose du câble et son raccordement seront effectués pour mi-2011 afin de pouvoir recevoir une première machine dès la fin de cette année-là dans le cadre de projets retenus et co-financés par l'ADEME suite à son AMI « Fonds démonstrateurs : Energies marines ».

Si le projet a pour vocation première d'aider à l'émergence de nouvelles technologies de conversion de l'énergie des vagues, il est possible que ces infrastructures soient mises à contribution pour développer l'éolien flottant. A plus court terme, il sera néanmoins et dès l'été 2010, pleinement disponible pour des activités scientifiques et de développement industriel dans le domaine de l'instrumentation, du naval et du côtier.

\section{Remerciements}

Le projet SEM-REV est porté par l'École Centrale de Nantes, encadré et financé par l'État, le Conseil régional des Pays de la Loire, le Conseil général de Loire-Atlantique et le Centre National de la Recherche Scientifique (CNRS). Le coût total de l'opération est de 5,8 millions d'euros (M€) dont 5 M€ sont financés par le CPER.

SEM-REV dispose d'un comité de pilotage formé par des représentants des organismes suivants :

- Le Secrétariat Général des Affaires Régionales

- Le Conseil régional des Pays de la Loire

- Le Conseil général de Loire-Atlantique

- Le CNRS

- L'École Centrale de Nantes

\section{Références}

HUERTAS-OLIVARES C., NEUMANN F., SARMENTO A. (2007). Environmental management recommendations for the wave energy Portuguese Pilot Zone. Proc. 7th European Wave and Tidal Energy Conference, Porto, Portugal.

KERBIRIOU M-A, PREVOSTO M., MAISONDIEU C., CLEMENT A.H., BABARIT A. (2007). Influence of sea-states description on wave energy production assessment. Proc.7th European Wave and Tidal Energy Conference, Porto, Portugal.

MOUSLIM H., BABARIT A., JORDANA N. (2008). Project Development of a Wave Energy Test Site in the French Atlantic Coast. Proc. 2nd International Conference on Ocean Energy, Brest, France. 